\title{
On Symbolic Ultrametrics, Cotree Representations, and Cograph Edge Decompositions and Partitions
}

\author{
Marc Hellmuth \\ Center for Bioinformatics, Saarland University, \\ Building E 2.1, D-66041 Saarbrücken, Germany \\ mhellmuth@bioinf .uni-sb.de \\ Nicolas Wieseke \\ Parallel Computing and Complex Systems Group, \\ Department of Computer Science, Leipzig University, \\ Augustusplatz 10, D-04109 Leipzig, Germany \\ wieseke@informatik.uni-leipzig.de
}

June 11, 2021

\begin{abstract}
Symbolic ultrametrics define edge-colored complete graphs $K_{n}$ and yield a simple tree representation of $K_{n}$. We discuss, under which conditions this idea can be generalized to find a symbolic ultrametric that, in addition, distinguishes between edges and non-edges of arbitrary graphs $G=(V, E)$ and thus, yielding a simple tree representation of $G$. We prove that such a symbolic ultrametric can only be defined for $G$ if and only if $G$ is a so-called cograph. A cograph is uniquely determined by a so-called cotree. As not all graphs are cographs, we ask, furthermore, what is the minimum number of cotrees needed to represent the topology of $G$. The latter problem is equivalent to find an optimal cograph edge $k$-decomposition $\left\{E_{1}, \ldots, E_{k}\right\}$ of $E$ so that each subgraph $\left(V, E_{i}\right)$ of $G$ is a cograph. An upper bound for the integer $k$ is derived and it is shown that determining whether a graph has a cograph 2-decomposition, resp., 2-partition is NP-complete.
\end{abstract}

\section{Introduction}

Given an arbitrary edge-colored complete graph $K_{n}=(V, E)$ on $n$ vertices, Böcker and Dress 4 asked, whether there is a tree representation of this $K_{n}$, i.e., a rooted tree $T=(W, F)$ with leaf set $V$ together with a labeling $t$ of the non-leaf vertices in $W \backslash V$ so that the least common ancestor lca $(x, y)$ of 
distinct leaves $x$ and $y$ is labeled with the respective color of the edge $[x, y] \in E$. The pair $(T, t)$ is then called symbolic representation of the edge-colored graph $K_{n}$. The authors showed, that there is a symbolic representation $(T, t)$ if and only if the map $\delta$ that assigns colors or symbols to the edges in $E$ fulfills the properties of a so-called symbolic ultrametric [4. Such maps are crucial for the characterization of relationships between genes or proteins, so-called orthology relations 13, 14, 15, that lie at the heart of many phylogenomic studies.

Inspired by the work of Böcker and Dress, we address the following problem: Does there exist, for an arbitrary undirected graph $G=(V, E)$ a symbolic ultrametric $\delta: V \times V \rightarrow M$ and thus, a symbolic representation $(T, t)$ of $G$ so that one can distinguish between edges and non-edges of $G$ ? In other words, we ask for a coloring $\delta$ of the edges $[x, y] \in E$, as well as the non-edges $[x, y] \notin E$, so that the topology of $G$ can be displayed by a rooted vertex-labeled tree $(T, t)$ s.t. for all distinct vertices $x, y \in V$ the labeling of the lowest common ancestor lca $(x, y)$ is equal to $\delta(x, y)$. The first result of this contribution provides that such a symbolic ultrametric can only be defined for $G$ if and only if $G$ is a cograph. This, in particular, establishes another new characterization of cographs.

Cographs are characterized by the absence of induced paths $P_{4}$ on four vertices. Moreover, Lerchs [16, 17] showed that each cograph $G=(V, E)$ is associated with a unique rooted tree $T(G)$, called cotree. Obviously, not all graphs are cographs and thus, don't have a cotree representation. Therefore, we ask for the minimum number of cotrees that are needed to represent the structure of a given graph $G=(V, E)$ in an unambiguous way. As it will turn out, this problem is equivalent to find a decomposition $\Pi=\left\{E_{1}, \ldots, E_{k}\right\}$ of $E$ (the elements of $\Pi$ need not necessarily be disjoint) for the least integer $k$, so that each subgraph $G_{i}=\left(V, E_{i}\right), 1 \leq i \leq k$ is a cograph. Such a decomposition is called cograph edge $k$-decomposition, or cograph $k$-decomposition, for short. If the elements of $\Pi$ are in addition pairwise disjoint, we call $\Pi$ a cograph $k$-partition. We will prove that finding the least integer $k \geq 2$ so that $G$ has a cograph $k$-decomposition or a cograph $k$-partition is an NP-hard problem. Moreover, upper bounds for the integer $k$ for any cograph $k$-decomposition are derived. These findings complement results known about so-called cograph vertex partitions [1, 11, 10, 26].

\section{Basic Definitions}

Graph. In what follows, we consider undirected simple graphs $G=(V, E)$ with vertex set $V(G)=V$ and edge set $E(G)=E \subseteq\left(\begin{array}{c}V \\ 2\end{array}\right)$. The complement graph $G^{c}=\left(V, E^{c}\right)$ of $G=(V, E)$, has edge set $E^{c}=\left(\begin{array}{c}V \\ 2\end{array}\right) \backslash E$. The graph $K_{|V|}=(V, E)$ with $E=\left(\begin{array}{c}V \\ 2\end{array}\right)$ is called complete graph. A graph $H=(W, F)$ is an induced subgraph of $G=(V, E)$, if $W \subseteq V$ and all edges $[x, y] \in E$ with $x, y \in W$ are contained in $F$. The degree $\operatorname{deg}(v)=|\{e \in E \mid v \in e\}|$ of a vertex $v \in V$ is defined as the number of edges that contain $v$. The maximum degree of a graph is denoted with $\Delta$. 
Rooted Tree. A connected graph $T$ is a tree, if $T$ does not contain cycles. A vertex of a tree $T$ of degree one is called a leaf of $T$ and all other vertices of $T$ are called inner vertices. The set of inner vertices of $T$ is denoted by $V^{0}$. A rooted tree $T=(V, E)$ is a tree that contains a distinguished vertex $\rho_{T} \in V$ called the root. The first inner vertex $\operatorname{lca}_{T}(x, y)$ that lies on both unique paths from distinct leaves $x$, resp., $y$ to the root, is called most recent common ancestor of $x$ and $y$. If there is no danger of ambiguity, we will write lca $(x, y)$ rather then $\operatorname{lca}_{T}(x, y)$.

Symbolic Ultrametric and Symbolic Representation. In what follows, the set $M$ will always denote a non-empty finite set, the symbol $\odot$ will always denote a special element not contained in $M$, and $M^{\odot}:=M \cup\{\odot\}$. Now, suppose $X$ is an arbitrary non-empty set and $\delta: X \times X \rightarrow M^{\odot}$ a map. We call $\delta$ a symbolic ultrametric if it satisfies the following conditions:

(U0) $\delta(x, y)=\odot$ if and only if $x=y$;

(U1) $\delta(x, y)=\delta(y, x)$ for all $x, y \in X$, i.e. $\delta$ is symmetric;

(U2) $|\{\delta(x, y), \delta(x, z), \delta(y, z)\}| \leq 2$ for all $x, y, z \in X$; and

(U3) there exists no subset $\{x, y, u, v\} \in\left(\begin{array}{c}X \\ 4\end{array}\right)$ such that $\delta(x, y)=\delta(y, u)=$ $\delta(u, v) \neq \delta(y, v)=\delta(x, v)=\delta(x, u)$.

Now, suppose that $T=(V, E)$ is a rooted tree with leaf set $X$ and that $t: V \rightarrow$ $M^{\odot}$ is a map such that $t(x)=\odot$ for all $x \in X$. To the pair $(T, t)$ we associate the map $d_{(T, t)}$ on $X \times X$ by setting, for all $x, y \in X$,

$$
d_{(T, t)}: X \times X \rightarrow M^{\odot} ; d_{(T, t)}(x, y)=t\left(\operatorname{lca}_{T}(x, y)\right) .
$$

Clearly this map is symmetric and satisfies (U0). We call the pair $(T, t)$ a symbolic representation of a map $\delta: X \times X \rightarrow M^{\odot}$, if $\delta(x, y)=d_{(T, t)}(x, y)$ holds for all $x, y \in X$. For a subset $W \subseteq X \times X$ we denote with $\delta(W)$ the restriction of $\delta$ to the set $W$.

Cographs and Cotrees. Complement-reducible graph, cographs for short, are defined as the class of graphs formed from a single vertex under the closure of the operations of union and complementation, namely: (i) a single-vertex graph is a cograph; (ii) the disjoint union of cographs is a cograph; (iii) the complement of a cograph is a cograph. Alternatively, a cograph can be defined as a $P_{4}$-free graph (i.e. a graph such that no four vertices induce a subgraph that is a path of length 3 ), although there are a number of equivalent characterizations of such graphs (see e.g. 6] for a survey). It is well-known in the literature concerning cographs that, to any cograph $G$, one can associate a canonical cotree $T(G)=(V, E)$. This is a rooted tree, leaf set equal to the vertex set $V(G)$ of $G$ and inner vertices that represent so-called "join" and "union" operations together with a labeling map $t: V^{0} \rightarrow\{0,1\}$ such that for all $[x, y] \in E(G)$ it holds that $t(\operatorname{lca}(x, y))=1$, and $t(v) \neq t\left(w_{i}\right)$ for all $v \in V^{0}$ and all children $w_{1}, \ldots, w_{k} \in V^{0}$ of $v$, (cf. [8]).

Cograph $k$-Decomposition and Partition, and Cotree Representation. Let $G=$ $(V, E)$ be an arbitrary graph. A decomposition $\Pi=\left\{E_{1}, \ldots E_{k}\right\}$ of $E$ is a 
called (cograph) $k$-decomposition, if each subgraph $G_{i}=\left(V, E_{i}\right), 1 \leq i \leq k$ of $G$ is a cograph. We call $\Pi$ a (cograph) $k$-partition if $E_{i} \cap E_{j}=\emptyset$, for all distinct $i, j \in\{1, \ldots, k\}$. A $k$-decomposition $\Pi$ is called optimal, if $\Pi$ has the least number $k$ of elements among all cograph decompositions of $G$. Clearly, for a cograph only k-decompositions with $k=1$ are optimal. A $k$-decomposition $\Pi=\left\{E_{1}, \ldots E_{k}\right\}$ is coarsest, if no elements of $\Pi$ can be unified, so that the resulting decomposition is a cograph $l$-decomposition, with $l<k$. In other words, $\Pi$ is coarsest, if for all subsets $I \subseteq\{1, \ldots, k\}$ with $|I|>1$ it holds that $\left(V, \cup_{i \in I} E_{i}\right)$ is not a cograph. Thus, every optimal $k$-decomposition is also always a coarsest one.

A graph $G=(V, E)$ is represented by a set of cotrees $\mathbb{T}=\left\{T_{1}, \ldots, T_{k}\right\}$, each $T_{i}$ with leaf set $V$, if and only if for each edge $[x, y] \in E$ there is a tree $T_{i} \in \mathbb{T}$ with $t\left(\operatorname{lca}_{T_{i}}(x, y)\right)=1$.

The Cartesian (Graph) Product $G \square H$ has vertex set $V(G \square H)=V(G) \times V(H)$; two vertices $\left(g_{1}, h_{1}\right),\left(g_{2}, h_{2}\right)$ are adjacent in $G \square H$ if $\left[g_{1}, g_{2}\right] \in E(G)$ and $h_{1}=$ $h_{2}$, or $\left[h_{1}, h_{2}\right] \in E(H)$ and $g_{1}=g_{2}$. It is well-known that the Cartesian product is associative, commutative and that the single vertex graph $K_{1}$ serves as unit element $\left[12\right.$. Thus, the product $\square_{i=1}^{n} G_{i}$ of arbitrary many factors $G_{1}, \ldots, G_{n}$ is well-defined. For a given product $\square_{i=1}^{n} G_{i}$, we define the $G_{i}$-layer $G_{i}^{w}$ of $G$ (through vertex $w$ that has coordinates $\left(w_{1}, \ldots, w_{n}\right)$ ) as the induced subgraph with vertex set $V\left(G_{i}^{w}\right)=\left\{v=\left(v_{1}, \ldots, v_{n}\right) \in \times_{i=1}^{n} V\left(G_{i}\right) \mid v_{j}=w_{j}\right.$, for all $j \neq$ $i$. Note, $G_{i}^{w}$ is isomorphic to $G_{i}$ for all $1 \leq i \leq n, w \in V\left(\square_{i=1}^{n} G_{i}\right)$. The $n$-cube $Q_{n}$ is the Cartesian product $\square_{i=1}^{n} K_{2}$.

\section{$3 \quad$ Symbolic Ultrametrics}

Symbolic ultrametrics and respective representations as event-labeled trees, have been first characterized by Böcker and Dress [4].

Theorem 3.1 ([4, 13]). Suppose $\delta: V \times V \rightarrow M^{\odot}$ is a map. Then there is a symbolic representation of $\delta$ if and only if $\delta$ is a symbolic ultrametric. Furthermore, this representation can be computed in polynomial time.

Let $\delta: V \times V \rightarrow M^{\odot}$ be a map satisfying Properties (U0) and (U1). For each fixed $m \in M$, we define an undirected graph $G_{m}:=G_{m}(\delta)=\left(V, E_{m}\right)$ with edge set

$$
E_{m}=\{\{x, y\} \mid \delta(x, y)=m, x, y \in V\} .
$$

Thus, the map $\delta$ can be considered as an edge coloring of a complete graph $K_{|V|}$, where each edge $[x, y]$ obtains color $\delta(x, y)$. Hence, $G_{m}$ denotes the subgraph of the edge-colored graph $K_{|V|}$, that contains all edges colored with $m \in M$. The following result establishes the connection between symbolic ultrametrics and cographs.

Theorem $3.2([13])$. Let $\delta: V \times V \rightarrow M^{\odot}$ be a map satisfying Properties (U0) and (U1). Then $\delta$ is a symbolic ultrametric if and only if 
(U2') For all $\{x, y, z\} \in\left(\begin{array}{l}V \\ 3\end{array}\right)$ there is an $m \in M$ such that $E_{m}$ contains two of the three edges $\{x, y\},\{x, z\}$, and $\{y, z\}$.

(U3') $G_{m}$ is a cograph for all $m \in M$.

Assume now, we have given an arbitrary subgraph $G=(V, E) \subseteq K_{|V|}$. Let $\delta$ be a map defined on $V \times V$ so that edges $e \in E$ obtain a different color then the non-edges $e \in E\left(K_{|V|}\right) \backslash E$ of $G$. The questions then arises, whether such a map fulfilling the properties of symbolic ultrametric can be defined and thus, if there is tree representation $(T, t)$ of $G$. Of course, this is possible only if $\delta$ restricted to $E$, resp., $E^{c}$ is a symbolic ultrametric, while it also a symbolic ultrametric on the complete graph $K_{|V|}=\left(V, E \cup E^{c}\right)$. The next theorem answers the latter question and, in addition, provides a new characterization of cographs.

Theorem 3.3. Let $G=(V, E)$ be an arbitrary (possibly disconnected) graph, $W=\{(x, y) \in V \times V \mid[x, y] \in E\}$ and $W^{c}=\{(x, y) \in V \times V \mid[x, y] \notin E\}$. There is a symbolic ultrametric $\delta: V \times V \rightarrow M^{\odot}$ s.t. $\delta(W) \cap \delta\left(W^{c}\right)=\emptyset$ if and only if $G$ is a cograph.

Proof. First assume that $G$ is a cograph. Set $\delta(x, x)=\odot$ for all $x \in V$ and set $\delta(x, y)=\delta(y, x)=1$ if $[x, y] \in E$ and, otherwise, to 0 . Hence, condition $(U 0)$ and $(U 1)$ are fulfilled. Moreover, by construction $|M|=2$ and thus, condition $\left(U 2^{\prime}\right)$ is trivially fulfilled. Furthermore, since $G_{1}(\delta)$ and its complement $G_{0}(\delta)$ are cographs, $\left(U 3^{\prime}\right)$ is satisfied. Theorem 3.2 implies that $\delta$ is a symbolic ultrametric.

Now, let $\delta: V \times V \rightarrow M^{\odot}$ be a symbolic ultrametric with $\delta(W) \cap \delta\left(W^{c}\right)=\emptyset$. Assume for contradiction that $G$ is not a cograph. Then $G$ contains an induced path $P_{4}=a-b-c-d$. Therefore, at least one edge $e$ of this path $P_{4}$ must obtain a color $\delta(e)$ different from the other two edges contained in this $P_{4}$, as otherwise $G_{\delta(e)}(\delta)$ is not a cograph and thus, $\delta$ is not a symbolic ultrametric (Theorem 3.2). For all such possible maps $\delta$ "subdividing" this $P_{4}$ we always obtain that two edges of at least one of the underlying paths $P_{3}=a-b-c$ or $b-c-d$ must have different colors. W.l.o.g. assume that $\delta(a, b) \neq \delta(b, c)$. Since $[a, c] \notin E$ and $\delta(W) \cap \delta\left(W^{c}\right)=\emptyset$ we can conclude that $\delta(a, c) \neq \delta(a, b)$ and $\delta(a, c) \neq \delta(b, c)$. But then condition $\left(U 2^{\prime}\right)$ cannot be satisfied, and Theorem 3.2 implies that $\delta$ is not a symbolic ultrametric.

The latter result implies, that there is no hope for finding a map $\delta$ for a graph $G$, that assigns symbols or colors to edges, resp., non-edges such that for $\delta$ (and hence, for $G$ ) there is a symbolic representation $(T, t)$, unless $G$ is already a cograph. In other words, every symbolic representation $(T, t)$ for an arbitrary graph $G$ (which only exists if $G$ is a cograph) is a cotree. However, this result does not come as a big surprise, as a cograph $G$ is characterized by the existence of a unique (up to isomorphism) cotree $(T, t)$ representing the topology of $G$. The (decision version of the) problem to edit a given graph $G$ into a cograph $G^{\prime}$, and thus, to find the closest graph $G^{\prime}$ that has a symbolic representation, is NP-complete [18, 19. In this contribution, we are interested in the following 


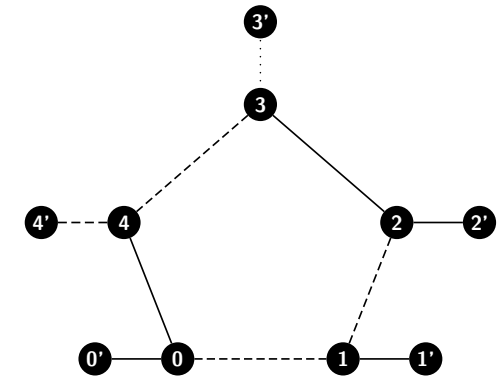

Figure 1: Full enumeration of all possibilities (which we leaf to the reader), shows that the depicted graph has no cograph 2-decomposition. However, it has a cograph 3-decomposition that is highlighted by dashed-lined, dotted and bold edges.

problem: What is the minimum number of cotrees that are needed to represent the topology of $G$ in an unambiguous way?

\section{Cotree Representation and Cograph $k$ - Decomposition}

Recollect, a graph $G=(V, E)$ is represented by a set of cotrees $\mathbb{T}=\left\{T_{1}, \ldots, T_{k}\right\}$, if and only if for each edge $[x, y] \in E$ there is a tree $T_{i} \in \mathbb{T}$ with $t\left(\operatorname{lca}_{T_{i}}(x, y)\right)=1$. Note, by definition, each cotree $T_{i}$ determines a subset $E_{i}=\{[x, y] \in E \mid$ $\left.t\left(\operatorname{lca}_{T_{i}}(x, y)\right)=1\right\}$ of $E$. Hence, the subgraph $\left(V, E_{i}\right)$ must be a cograph. Therefore, in order to find the minimum number of cotrees representing a graph $G$, we can equivalently ask for a decomposition $\Pi=\left\{E_{1}, \ldots, E_{k}\right\}$ of $E$ so that each subgraph $\left(V, E_{i}\right)$ is a cograph, where $k$ is the least integer among all cograph decompositions of $G$. Thus, we are dealing with the following two equivalent problems.

Problem. Cotree $k$-Representation

Input: $\quad$ Given a graph $G=(V, E)$ and an integer $k$.

Question: Can $G$ be represented by $k$ cotrees?

Problem. Cograph $k$-Decomposition

Input: $\quad$ Given a graph $G=(V, E)$ and an integer $k$.

Question: Is there a cograph k-decomposition of $G$ ?

Clearly, any cograph has an optimal 1-decomposition, while for cycles of length $>4$ or paths $P_{4}$ there is always an optimal cograph 2-decomposition. However, there are examples of graphs that do not have a 2-decomposition, see Figure 1. To derive an upper bound for the integer $k$ s.t. there is a cograph $k$-decomposition for arbitrary graphs, the next theorem is given. 
Theorem 4.1. For every graph $G$ with maximum degree $\Delta$ there is a cograph $k$-decomposition with $1 \leq k \leq \Delta+1$ that can be computed in $O(|V||E|+\Delta(|V|+$ $|E|))$ time. Hence, any graph can be represented by at most $\Delta+1$ cotrees.

Proof. Consider a proper edge-colorings $\varphi: E \rightarrow\{1, \ldots, k\}$ of $G$, i.e., an edge coloring such that no two incident edges obtain the same color. Any proper edge-coloring using $k$ colors yields a cograph $k$-partition $\Pi=\left\{E_{1}, \ldots, E_{k}\right\}$ where $E_{i}=\{e \in E \mid \varphi(e)=i\}$, because any connected component in $G_{i}=\left(V, E_{i}\right)$ is an edge and thus, no $P_{4}$ 's are contained in $G_{i}$. Vizing's Theorem [25] implies that for each graph there is a proper edge-coloring using $k$ colors with $\Delta \leq k \leq \Delta+1$.

An proper edge-coloring using at most $\Delta+1$ colors can be computed with the Misra-Gries-algorithm in $O(|V||E|)$ time [20]. Since the (at most $\Delta+1$ ) respective cotrees can be constructed in linear-time $O(|V|+|E|)[9$, we derive the runtime $O(|V||E|+\Delta(|V|+|E|))$.

Obviously, any optimal $k$-decomposition must also be a coarsest $k$ decomposition, while the converse is in general not true, see Fig.2. The partition $\Pi=\left\{E_{1}, \ldots, E_{k}\right\}$ obtained from a proper edge-coloring is usually not a coarsest one, as possibly $\left(V, E_{J}\right)$ is a cograph, where $E_{J}=\cup_{i \in J} E_{i}$ and $J \subseteq\{1, \ldots, l\}$. A graph having an optimal cograph $\Delta$-decomposition is shown in Fig. 1 Thus, the derived bound $\Delta+1$ is almost sharp. Nevertheless, we assume that this bound can be sharpened:

Conjecture 1. For every graph $G$ with maximum degree $\Delta$ there is a cograph $\Delta$-decomposition.

However, there are examples of non-cographs containing many induced $P_{4}$ 's that have a cograph $k$-decomposition with $k \ll \Delta+1$, which implies that any optimal $k$-decomposition of those graphs will have significantly less elements than $\Delta+1$, see the following examples.

Example 1. Consider the graph $G=(V, E)$ with vertex set $V=\{1, \ldots, k\} \cup$ $\{a, b\}$ and $E=\{[i, j] \mid i, j \in\{1, \ldots, k\}, i \neq j\} \cup\{[k, a],[a, b]\}$. The graph $G$ is not a cograph, since there are induced $P_{4}$ 's of the form $i-k-a-b$, $i \in\{1, \ldots, k-1\}$. On the other hand, the subgraph $H=(V, E \backslash\{[k, a]\})$ has two connected components, one is isomorphic to the complete graph $K_{k}$ on $k$ vertices and the other to the complete graph $K_{2}$. Hence, $H$ is a cograph. Therefore, $G$ has a cograph 2-partition $\{E \backslash\{[k, a]\},\{[k, a]\}\}$, independent from $k$ and thus, independent from the maximum degree $\Delta=k$.

Example 2. Consider the 2n-dimensional hypercube $Q_{2 n}=(V, E)$ with maximum degree $2 n$. We will show that this hypercube has a coarsest cograph $n$-partition $\Pi=\left\{E_{1}, \ldots, E_{n}\right\}$, which implies that for any optimal cograph $k$ decomposition of $Q_{2 n}$ we have $k \leq \Delta / 2$.

We construct now a cograph $n$-partition of $Q_{2 n}$. Note, $Q_{2 n}=\square_{i=1}^{2 n} K_{2}=$ $\square_{i=1}^{n}\left(K_{2} \square K_{2}\right)=\square_{i=1}^{n} Q_{2}$. In order to avoid ambiguity, we write $\square_{i=1}^{n} Q_{2}$ as $\square_{i=1}^{n} H_{i}, H_{i} \simeq Q_{2}$ and assume that $Q_{2}$ has edges [0,1], [1,2], [2,3], $[3,0]$. The cograph n-partition of $Q_{2 n}$ is defined as $\Pi=\left\{E_{1}, \ldots, E_{n}\right\}$, where $E_{i}=$ 


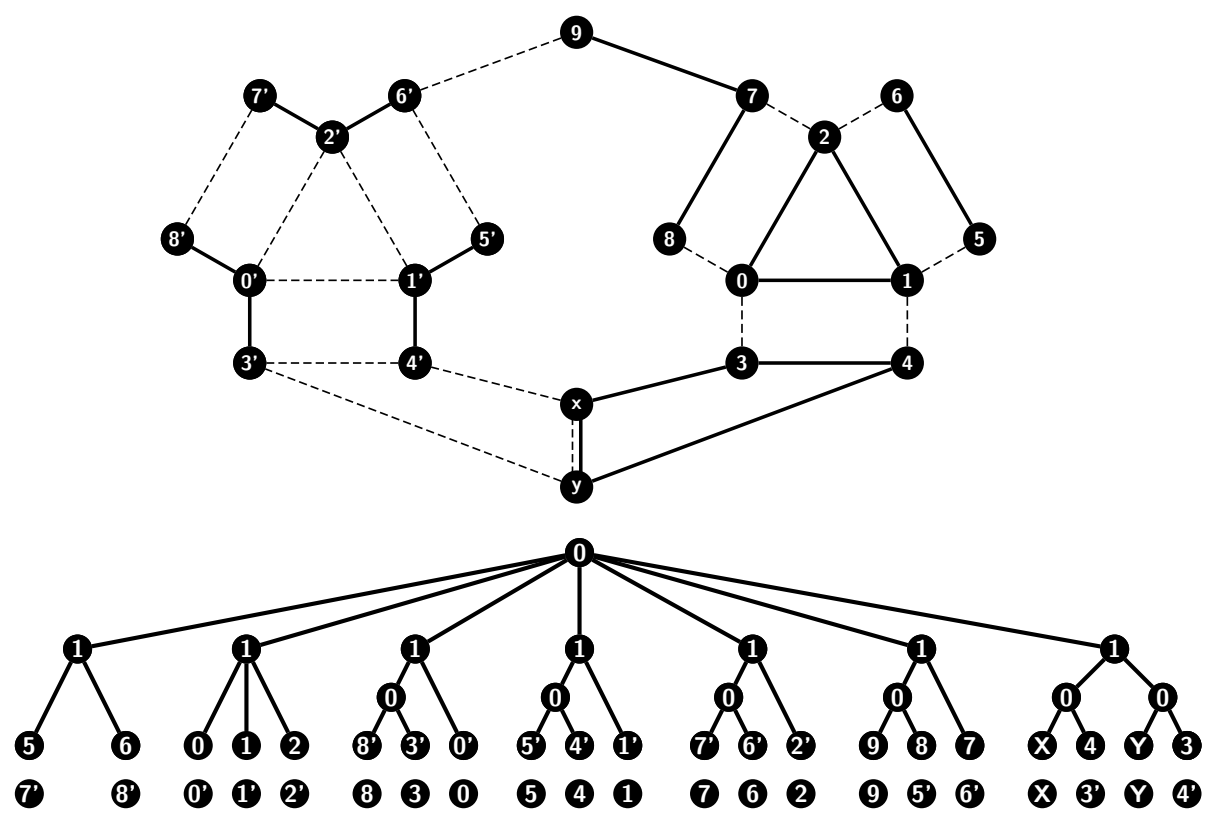

Figure 2: The shown (non-co)graph $G$ has a 2-decomposition $\Pi=\left\{E_{1}, E_{2}\right\}$. Edges in the different elements $E_{1}$ and $E_{2}$ are highlighted by dashed and solid edges, respectively. Thus, two cotrees, shown in the lower part of this picture, are sufficient to represent the structure of $G$. The two cotrees are isomorphic, and thus, differ only in the arrangement of their leaf sets. For this reason, we only depicted one cotree with two different leaf sets. Note, $G$ has no 2-partition, but a coarsest 3-partition. The latter can easily be verified by application of the construction in Lemma 4.2

$\cup_{v \in V} E\left(H_{i}^{v}\right)$. In other words, the edge set of all $H_{i}$-layers in $Q_{2 n}$ constitute a single class $E_{i}$ in the partition for each $i$. Therefore, the subgraph $G=\left(V, E_{i}\right)$ consists of $n$ connected components, each component is isomorphic to the square $Q_{2}$. Hence, $G_{i}=\left(V, E_{i}\right)$ is a cograph.

Assume for contradiction that $\Pi=\left\{E_{1}, \ldots, E_{n}\right\}$ is not a coarsest partition. Then there are distinct classes $E_{i}, i \in I \subseteq\{1, \ldots, n\}$ such that $G_{I}=\left(V, \cup_{i \in I} E_{i}\right)$ is a cograph. W.l.o.g. assume that $1,2 \in I$ and let $v=$ $(0, \ldots, 0) \in V$. Then, the subgraph $H_{1}^{v} \cup H_{2}^{v} \subseteq Q_{2 n}$ contains a path $P_{4}$ with edges $[x, v] \in E\left(H_{1}^{v}\right)$ and $[v, a],[a, b] \in E\left(H_{2}^{v}\right)$, where $x=(1,0, \ldots, 0), a=(0,1,0 \ldots, 0)$ and $b=(0,2,0 \ldots, 0)$. By definition of the Cartesian product, there are no edges connecting $x$ with $a$ or $b$ or $v$ with $b$ in $Q_{2 n}$ and thus, this path $P_{4}$ is induced. As this holds for all subgraphs $H_{i}^{v} \cup H_{j}^{v}(i, j \in I$ distinct) and thus, in particular for the graph $G_{I}$ we can conclude that classes of $\Pi$ cannot be combined. Hence $\Pi$ is a coarsest cograph n-partition.

Because of the results of computer-aided search for $n$-1-partitions and decompositions of hypercubes $Q_{2 n}$ we are led to the following conjecture: 
Conjecture 2. Let $k \in \mathbb{N}$ and $k>1$. Then the $2 k$-cube has no cograph $k-1$ decomposition, i.e., the proposed $k$-partition of the hypercube $Q_{2 k}$ in Example 0 is also optimal.

The proof of the latter hypothesis would immediately verify the next conjecture.

Conjecture 3. For every $k \in \mathbb{N}$ there is a graph that has an optimal cograph $k$-decomposition.

Proving the last conjecture appears to be difficult. We wish to point out that there is a close relationship to the problem of finding pattern avoiding words, see e.g. [5, 7, 23, 22, 3, 22: Consider a graph $G=(V, E)$ and an ordered list $\left(e_{1}, \ldots, e_{m}\right)$ of the edges $e_{i} \in E$. We can associate to this list $\left(e_{1}, \ldots, e_{m}\right)$ a word $w=\left(w_{1}, \ldots, w_{m}\right)$. By way of example, assume that we want to find a valid cograph 2-decomposition $\left\{E_{1}, E_{2}\right\}$ of $G$ and that $G$ contains an induced $P_{4}$ consisting of the edges $e_{i}, e_{j}, e_{k}$. Hence, one has to avoid assignments of the edges $e_{i}, e_{j}, e_{k}$ to the single set $E_{1}$, resp., $E_{2}$. The latter is equivalent to find a binary word $\left(w_{1}, \ldots, w_{m}\right)$ such that $\left(w_{i}, w_{j}, w_{k}\right) \neq(X, X, X), X \in\{0,1\}$ for each of those induced $P_{4}$ 's. The latter can easily be generalized to find pattern avoiding words over an alphabet $\{1, \ldots, k\}$ to get a valid $k$-decomposition. However, to the authors knowledge, results concerning the counting of $k$-ary words avoiding forbidden patterns and thus, verifying if there is any such word (or equivalently a $k$-decomposition) are basically known for scenarios like: If $\left(p_{1}, \ldots p_{l}\right) \in\{1, \ldots, k\}^{l}$ (often $l<3$ ), then none of the words $w$ that contain a subword $\left(w_{i_{1}}, \ldots, w_{i_{l}}\right)=\left(p_{1}, \ldots p_{l}\right)$ with $i_{j+1}=i_{j}+1$ (consecutive letter positions) or $i_{j}<i_{k}$ whenever $j<k$ (order-isomorphic letter positions) is allowed. However, such findings are to restrictive to our problem, since we are looking for words, that have only on a few, but fixed positions of non-allowed

patterns. Nevertheless, we assume that results concerning the recognition of pattern avoiding words might offer an avenue to solve the latter conjectures.

\subsection{NP-completeness and NP-hardness Results}

We are now in the position to prove the NP-completeness of CotreE 2Representation and Cotree 2-Decomposition. These results allow to show that the problem of determining whether there is cograph 2-partition is NP-complete, as well.

We start with two lemmata concerning cograph 2-decompositions of the graphs shown in Fig. 3 and 4

Lemma 4.2. For the literal and extended literal graph in Figure 3 every cograph 2-decomposition is a uniquely determined cograph 2-partition.

In particular, in every cograph 2-partition $\left\{E_{1}, E_{2}\right\}$ of the extended literal graph, the edges of the triangle $(0,1,2)$ must be entirely contained in one $E_{i}$ and the pending edge [6,9] must be in the same edge set $E_{i}$ as the edges of the of the triangle. Furthermore, the edges $[9,10]$ and $[9,11]$ must be contained in $E_{j}$, $i \neq j$. 
Proof. It is easy to verify that the given cograph 2-partition $\left\{E_{1}, E_{2}\right\}$ in Fig. 3 fulfills the conditions and is correct, since $G=\left(V, E_{1}\right)$ and $G=\left(V, E_{2}\right)$ do not contain induced $P_{4}$ 's and are, thus, cographs. We have to show that it is also unique.

Assume that there is another cograph 2-decomposition $\left\{F_{1}, F_{2}\right\}$. Note, for any cograph 2-decomposition $\left\{F_{1}, F_{2}\right\}$ it must hold that two incident edges in the triangle $(0,1,2)$ are contained in one of the sets $F_{1}$ or $F_{2}$. W.l.o.g. assume that $[0,1],[0,2] \in F_{1}$.

Assume first that $[1,2] \notin F_{1}$. In this case, because of the paths $P_{4}=$ $6-2-0-1$ and $P_{4}=2-0-1-5$ it must hold that $[2,6],[1,5] \notin F_{1}$ and thus, $[2,6],[1,5] \in F_{2}$. However, in this case and due to the paths $P_{4}=6-2-1-4$ and $2-0-1-4$ the edge $[1,4]$ can neither be contained in $F_{1}$ nor in $F_{2}$, a contradiction. Hence, $[1,2] \in F_{1}$.

Note, the square $S_{1256}$ induced by vertices $1,2,5,6$ cannot have all edges in $F_{1}$, as otherwise the subgraph $\left(V, F_{1}\right)$ would contain the induced $P_{4}=6-5-$ $1-0$. Assume that $[1,5] \in F_{1}$. As not all edges $S_{1256}$ are contained in $F_{1}$, at least one of the edges $[5,6]$ and $[2,6]$ must be contained in $F_{2}$. If only one of the edges $[5,6]$, resp., $[2,6]$ is contained in $F_{2}$, we immediately obtain the induced $P_{4}=6-2-1-5$, resp., $6-5-1-2$ in $\left(V, F_{1}\right)$ and therefore, both edges $[5,6]$ and $[2,6]$ must be contained in $F_{2}$. But then the edge $[2,7]$ can neither be contained in $F_{1}$ (due to the induced $P_{4}=5-1-2-7$ ) nor in $F_{2}$ (due to the induced $\left.P_{4}=5-6-2-7\right)$, a contradiction. Hence, $[1,5] \notin F_{1}$ and thus, $[1,5] \in F_{2}$ for any 2-decomposition. By analogous arguments and due to symmetry, all edges $[0,3],[0,8],[1,4],[2,6],[2,7]$ are contained in $F_{2}$, but not in $F_{1}$.

Moreover, due to the induced $P_{4}=7-2-6-5$ and since $[2,6],[2,7] \in F_{2}$, the edge $[5,6]$ must be in $F_{1}$ and not in $F_{2}$. By analogous arguments and due to symmetry, it holds that $[3,4],[7,8] \in F_{1}$ and $[3,4],[7,8] \notin F_{2}$. Finally, none of the edges of the triangle $(0,1,2)$ can be contained in $F_{2}$, as otherwise, we obtain an induced $P_{4}$ in $\left(V, F_{2}\right)$. Taken together, any 2-decomposition of the literal graph must be a partition and is unique.

Consider now the extended literal graph in Figure 3. As this graph contains the literal graph as induced subgraph, the unique 2-partition of the underlying literal graph is determined as by the preceding construction. Due to the path $P_{4}=7-2-6-9$ with $[2,6],[2,7] \in F_{2}$ we can conclude that $[6,9] \notin F_{2}$ and thus $[6,9] \in F_{1}$. Since there are induced paths $P_{4}=5-6-9-y, y=10,11$ with $[5,6],[6,9] \in F_{1}$ we obtain that $[9,10],[9,11] \notin F_{1}$ and thus, $[9,10],[9,11] \in F_{2}$ for any 2-decomposition (which is in fact a 2-partition) of the extended literal graph, as claimed.

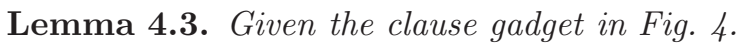

For any cograph 2-decomposition, all edges of exactly two of the triangles in the underlying three extended literal graphs must be contained in one $E_{i}$ and not in $E_{j}$, while the edges of the triangle of one extended literal graph must be in $E_{j}$ and not in $E_{i}, i \neq j$. 

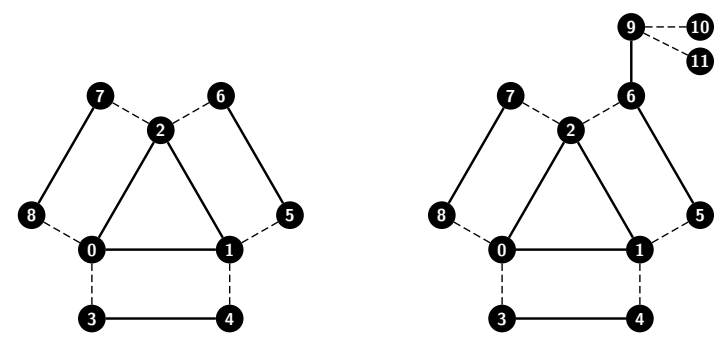

Figure 3: Left the literal graph and right the extended literal graph with unique corresponding cograph 2-partition (indicated by dashed and bold-lined edges) is shown.

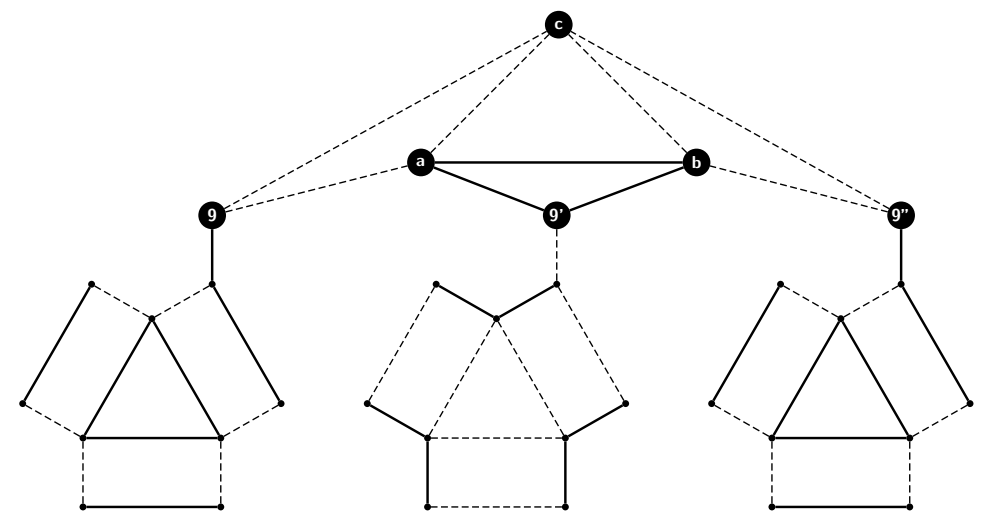

Figure 4: Shown is a clause gadget which consists of a triangle $(a, b, c)$ and three extended literal graphs (as shown in Fig. 3) with edges attached to $(a, b, c)$. A corresponding cograph 2-partition is indicated by dashed and bold-line edges.

Furthermore, for each cograph 2-decomposition exactly two of the edges e, $e^{\prime}$ of the triangle $(a, b, c)$ must be in one $E_{i}$ while the other edge $f$ is in $E_{j}$ but not in $E_{i}, j \neq i$. The cograph 2-decomposition can be chosen so that in addition $e, e^{\prime} \notin E_{j}$, resulting in a cograph 2-partition of the clause gadget.

Proof. It is easy to verify that the given cograph 2-partition in Fig. 4 fulfills the conditions and is correct, as $G=\left(V, E_{1}\right)$ and $G=\left(V, E_{2}\right)$ are cographs.

As the clause gadget contains the literal graph as induced subgraph, the unique 2-partition of the underlying literal graph is determined as by the construction given in Lemma 4.2. Thus, each edge of the triangle in each underlying literal graph is contained in either one of the sets $E_{1}$ or $E_{2}$. Assume that edges of the triangles in the three literal gadgets are all contained in the same set, say $E_{1}$. Then, Lemma 4.2 implies that $[9, a],[9, c],\left[9^{\prime}, a\right],\left[9^{\prime}, b\right],\left[9^{\prime \prime}, b\right],\left[9^{\prime \prime}, c\right] \in E_{1}$ and none of them is contained in $E_{2}$. Since there are induced $P_{4}$ 's: $9-a-b-9^{\prime \prime}$, $9^{\prime}-a-c-9^{\prime \prime}$ and $9-c-b-9^{\prime}$, the edges $[a, b],[a, c],[b, c]$ cannot be contained in $E_{1}$, and thus must be in $E_{2}$. However, this is not possible, since then we 
would have the induced paths $P_{4}=9-a-9^{\prime}-b$ in the subgraph $\left(V, E_{1}\right)$ a contradiction. Thus, the edges of the triangle of exactly one literal gadget must be contained in a different set $E_{i}$ than the edges of the other triangles in the other two literal gadgets. W.l.o.g. assume that the 2-decomposition of the underlying literal gadgets is given as in Fig. 4, and identify bold-lined edges with $E_{1}$ and dashed edges with $E_{2}$.

It remains to show that this 2-decomposition of the underlying three literal gadgets determines which of the edges of triangle $(a, b, c)$ are contained in which of the sets $E_{1}$ and $E_{2}$. Due to the induced path $9-a-b-9^{\prime \prime}$ and since $[9, a],\left[9^{\prime \prime}, b\right] \in E_{2}$, the edge $[a, b]$ cannot be contained in $E_{2}$ and thus, is contained in $E_{1}$. Moreover, if $[b, c] \notin E_{2}$, then then there is an induced path $P_{4}=$ $b-9^{\prime \prime}-c-9$ in the subgraph $\left(V, E_{2}\right)$, a contradiction. Hence, $[b, c] \in E_{2}$ and by analogous arguments, $[a, c] \in E_{2}$. If $[b, c] \notin E_{1}$ and $[a, c] \notin E_{1}$, then we obtain a cograph 2-partition. However, it can easily be verified that there is still a degree of freedom and $[a, c],[b, c] \in E_{1}$ is allowed for a valid cograph 2-decomposition.

We are now in the position to prove NP-completeness of COGRAPH 2PARTITION by reduction from the following problem.

Problem. Monotone NAE 3-SAT

Input: $\quad$ Given a set $U$ of Boolean variables and a set of clauses $\psi=\left\{C_{1}, \ldots, C_{m}\right\}$ over $U$ such that for all $i=1, \ldots, m$ it holds that $\left|C_{i}\right|=3$ and $C_{i}$ contains no negated variables.

Question: Is there a truth assignment to $\psi$ such that in each $C_{i}$ not all three literals are set to true?

Theorem 4.4 (24, 21]). Monotone NAE 3-SAT is NP-complete.

Theorem 4.5. Cograph 2-Decomposition, and thus, Cotree 2REPRESENTATION is NP-complete.

Proof. Given a graph $G=(V, E)$ and cograph 2-decomposition $\left\{E_{1}, E_{2}\right\}$, one can verify in linear time whether $\left(V, E_{i}\right)$ is a cograph 9]. Hence, Cograph 2-PARTition $\in$ NP.

We will show by reduction from Monotone NAE 3-SAT that CogRAPH 2Decomposition is NP-hard. Let $\psi=\left(C_{1}, \ldots, C_{m}\right)$ be an arbitrary instance of Monotone NAE 3-SAT. Each clause $C_{i}$ is identified with a triangle $\left(a_{i}, b_{i}, c_{i}\right)$. Each variable $x_{j}$ is identified with a literal graph as shown in Fig. 3 (left) and different variables are identified with different literal graphs. Let $C_{i}=$ $\left(x_{i_{1}}, x_{i_{2}}, x_{i_{3}}\right)$ and $G_{i_{1}}, G_{i_{2}}$ and $G_{i_{3}}$ the respective literal graphs. Then, we extend each literal graph $G_{i_{j}}$ by adding an edge $\left[6,9_{i, j}\right]$. Moreover, we add to $G_{i_{1}}$ the edges $\left[9_{i, 1}, a_{i}\right],\left[9_{i, 1}, c_{i}\right]$, to $G_{i_{2}}$ the edges $\left[9_{i, 2}, a_{i}\right],\left[9_{i, 2}, b_{i}\right]$, to $G_{i_{3}}$ the edges $\left[9_{i, 3}, c_{i}\right],\left[9_{i, 3}, b_{i}\right]$. The latter construction connects each literal graph with the triangle $\left(a_{i}, b_{i}, c_{i}\right)$ of the respective clause $C_{i}$ in a unique way, see Fig. 4. We denote the clause gadgets by $\Psi_{i}$ for each clause $C_{i}$. We repeat this construction for all clauses $C_{i}$ of $\psi$ resulting in the graph $\Psi$. An illustrative example is given 


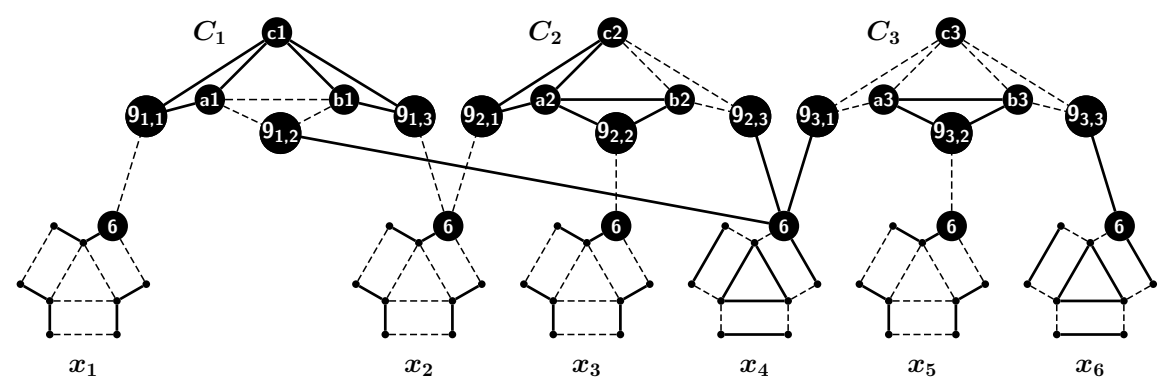

Figure 5: Shown is the graph $\Psi$ as constructed in the proof of Theorem 4.5 In particular, $\Psi$ reflects the NAE 3-SAT formula $\psi=\left\{C_{1}, C_{2}, C_{3}\right\}$ with clauses $C_{1}=\left(x_{1}, x_{4}, x_{2}\right), C_{2}=\left(x_{2}, x_{3}, x_{4}\right)$ and $C_{3}=\left(x_{4}, x_{5}, x_{6}\right)$. Different literals obtain the same truth assignment true or false, whenever the edges of the triangle in their corresponding literal gadget are contained in the same set $E_{i}$ of the cograph 2-partition, highlighted by dashed and bold-lined edges.

in Fig. [5. Clearly, this reduction can be done in polynomial time in the number $m$ of clauses.

We will show in the following that $\Psi$ has a cograph 2-decomposition (resp., a cograph 2-partition) if and only if $\psi$ has a truth assignment $f$.

Let $\psi=\left(C_{1}, \ldots, C_{m}\right)$ have a truth assignment. Then in each clause $C_{i}$ at least one of the literals $x_{i_{1}}, x_{i_{2}}, x_{i_{3}}$ is set to true and one to false. We assign all edges $e$ of the triangle in the corresponding literal graph $G_{i_{j}}$ to $E_{1}$, if $f\left(x_{i_{j}}\right)=$ true and to $E_{2}$, otherwise. Hence, each edge of exactly two of the triangles (one in $G_{i_{j}}$ and one in $G_{i_{j^{\prime}}}$ contained in one $E_{r}$ and not in $E_{s}$, while the edges of the other triangle in $G_{i_{j^{\prime \prime}}}, j^{\prime \prime} \neq j, j^{\prime}$ are contained in $E_{s}$ and not in $E_{r}, r \neq s$, as needed for a possible valid cograph 2-decomposition (Lemma 4.3). We now apply the construction of a valid 2-decomposition (or 2-partition) for each $\Psi_{i}$ as given in Lemma 4.3. starting with the just created assignment of edges contained in the triangles in $G_{i_{j}}, G_{i_{j^{\prime}}}$ and $G_{i_{j^{\prime \prime}}}$ to $E_{1}$ or $E_{2}$. In this way, we obtain a valid 2-decomposition (or 2-partition) for each subgraph $\Psi_{i}$ of $\Psi$. Thus, if there would be an induced $P_{4}$ in $\Psi$ with all edges belonging to the same set $E_{r}$, then this $P_{4}$ can only have edges belonging to different clause gadgets $\Psi_{k}, \Psi_{l}$. By construction, such a $P_{4}$ can only exist along different clause gadgets $\Psi_{k}$ and $\Psi_{l}$ only if $C_{k}$ and $C_{l}$ have a literal $x_{i}=x_{k_{m}}=x_{l_{n}}$ in common. In this case, Lemma 4.3 implies that the edges $\left[6,9_{k, m}\right]$ and $\left[6,9_{l, n}\right]$ in $\Psi_{i}$ must belong to the same set $E_{r}$. Again by Lemma 4.3. the edges $\left[9_{k, m}, y\right]$ and $\left[9_{k, m}, y^{\prime}\right], y, y^{\prime} \in\left\{a_{k}, b_{k}, c_{k}\right\}$ as well as the edges $\left[9_{l, n}, y\right]$ and $\left[9_{l, n}, y^{\prime}\right], y, y^{\prime} \in\left\{a_{l}, b_{l}, c_{l}\right\}$ must be in a different set $E_{s}$ than $\left[6,9_{k, m}\right]$ and $\left[6,9_{l, n}\right]$. Moreover, respective edges $[5,6]$ in $\Psi_{k}$, as well as in $\Psi_{l}$ (Fig. 3) must then be in $E_{r}$, i.e., in the same set as $\left[6,9_{k, m}\right]$ and $\left[6,9_{l, n}\right]$. However, in none of the cases it is possible to find an induced $P_{4}$ with all edges in the same set $E_{r}$ or $E_{s}$ along different clause gadgets. Hence, we obtain a valid cograph 2-decomposition, resp., cograph 2-partition of $\Psi$.

Now assume that $\Psi$ has a valid cograph 2-decomposition (or a 2-partition). 
Any variable $x_{i_{j}}$ contained in some clause $C_{i}=\left(x_{i_{1}}, x_{i_{2}}, x_{i_{3}}\right)$ is identified with a literal graph $G_{i_{j}}$. Each clause $C_{i}$ is, by construction, identified with exactly three literal graphs $G_{i_{1}}, G_{i_{2}}, G_{i_{3}}$, resulting in the clause gadget $\Psi_{i}$. Each literal graph $G_{i_{j}}$ contains exactly one triangle $t_{j}$. Since $\Psi_{i}$ is an induced subgraph of $\Psi$, we can apply Lemma 4.3 and conclude that for any cograph 2-decomposition (resp., 2-partition) all edges of exactly two of three triangles $t_{1}, t_{2}, t_{3}$ are contained in one set $E_{r}$, but not in $E_{s}$, and all edges of the other triangle are contained in $E_{s}$, but not in $E_{r}, s \neq r$. Based on these triangles we define a truth assignment $f$ to the corresponding literals: w.l.o.g. we set $f\left(x_{i}\right)=$ true if the edge $e \in t_{i}$ is contained in $E_{1}$ and $f\left(x_{i}\right)=$ false otherwise. By the latter arguments and Lemma 4.3 we can conclude that, given a valid cograph 2-partitioning, the so defined truth assignment $f$ is a valid truth assignment of the Boolean formula $\psi$, since no three different literals in one clause obtain the same assignment and at least one of the variables is set to true. Thus, CograPH 2-Decomposition is NP-complete

Finally, because Cograph 2-Decomposition and Cotree 2REPRESENTATION are equivalent problems, the NP-completeness of CotreE 2-REPRESENTATION follows.

As the proof of Theorem 4.5 allows us to use cograph 2-partitions in all proof steps, instead of cograph 2-decompositions, we can immediately infer the $\mathrm{NP}$-completeness of the following problem for $\mathrm{k}=2$, as well.

\section{Problem. Cograph $k$-PARTition}

Input: $\quad$ Given a graph $G=(V, E)$ and an integer $k$.

Question: Is there a Cograph k-Partition of $G$ ?

Theorem 4.6. Cograph 2-Partition is NP-complete.

As a direct consequence of the latter results, we obtain the following theorem.

Theorem 4.7. Let $G$ be a given graph that is not a cograph. The following three optimization problems to find the least integer $k>1$ so that there is a Cograph $k$-Partition, or a Cograph $k$-Decomposition, or a Cotree $k$-Representation for the graph $G$, are NP-hard.

\section{Acknowledgment}

This work was funded by the German Research Foundation (DFG) (Proj. No. MI439/14-1).

\section{References}

[1] D. Achlioptas. The complexity of g-free colourability. Discrete Mathemat$i c s, 165166(0): 21$ - 30, 1997. Graphs and Combinatorics. 
[2] A. Bernini, L. Ferrari, and R. Pinzani. Enumeration of some classes of words avoiding two generalized patterns of length three. arXiv preprint arXiv:0711.3387, 2007.

[3] S. Bilotta, E. Grazzini, E. Pergola, and V.GB. Morgagni. Counting binary words avoiding alternating patterns. Journal of Integer Sequences, 16(2):3, 2013.

[4] S. Böcker and A.W.M. Dress. Recovering symbolically dated, rooted trees from symbolic ultrametrics. Adv. Math., 138:105-125, 1998.

[5] P. Brändén and T. Mansour. Finite automata and pattern avoidance in words. Journal of Combinatorial Theory, Series A, 110(1):127 - 145, 2005.

[6] A. Brandstädt, V.B. Le, and J.P. Spinrad. Graph Classes: A Survey. SIAM Monographs on Discrete Mathematics and Applications. Soc. Ind. Appl. Math., Philadephia, 1999.

[7] A. Burstein and T. Mansour. Words restricted by patterns with at most 2 distinct letters. Electron. J. Combin. Number Theory, 9(2):1-16, 2002.

[8] D. G. Corneil, H. Lerchs, and L K Stewart Burlingham. Complement reducible graphs. Discr. Appl. Math., 3:163-174, 1981.

[9] D.G. Corneil, Y. Perl, and L.K. Stewart. A linear recognition algorithm for cographs. SIAM Journal on Computing, 14(4):926-934, 1985.

[10] P. Dorbec, M. Montassier, and P. Ochem. Vertex partitions of graphs into cographs and stars. Journal of Graph Theory, 75(1):75-90, 2014.

[11] J. Gimbel and J. Nesětril. Partitions of graphs into cographs. Electronic Notes in Discrete Mathematics, 11(0):705 - 721, 2002. The Ninth Quadrennial International Conference on Graph Theory, Combinatorics, Algorithms and Applications.

[12] R. Hammack, W. Imrich, and S. Klavžar. Handbook of Product graphs. CRC Press, Boca Raton, 2nd edition, 2011.

[13] M. Hellmuth, M. Hernandez-Rosales, K. T. Huber, V. Moulton, P. F. Stadler, and N. Wieseke. Orthology relations, symbolic ultrametrics, and cographs. Journal of Mathematical Biology, 66(1-2):399-420, 2013.

[14] M. Hellmuth, N. Wiesecke, H.P. Lenhof, M. Middendorf, and P.F. Stadler. Phylogenomics with paralogs. PNAS, 2015. (in press).

[15] M. Lafond and N. El-Mabrouk. Orthology and paralogy constraints: satisfiability and consistency. BMC Genomics, 15(Suppl 6):S12, 2014.

[16] H. Lerchs. On cliques and kernels. Technical report, Dept. of Comput. Sci. University of Toronto, 1971. 
[17] H. Lerchs. On the clique-kernel structure of graphs. Technical report, Dept. of Comput. Sci. University of Toronto, 1971.

[18] Y. Liu, J. Wang, J. Guo, and J. Chen. Cograph editing: Complexity and parametrized algorithms. In B. Fu and D. Z. Du, editors, COCOON 2011, volume 6842 of Lect. Notes Comp. Sci., pages 110-121, Berlin, Heidelberg, 2011. Springer-Verlag.

[19] Y. Liu, J. Wang, J. Guo, and J. Chen. Complexity and parameterized algorithms for cograph editing. Theoretical Computer Science, 461(0):45 54,2012 .

[20] J. Misra and David Gries. A constructive proof of vizing's theorem. Information Processing Letters, 41(3):131 - 133, 1992.

[21] B. M. Moret. The Theory of Computation. Addison-Wesley, 1997.

[22] L.K. Pudwell. Enumeration schemes for pattern-avoiding words and permutations. ProQuest, 2008.

[23] L.K. Pudwell. Enumeration schemes for words avoiding patterns with repeated letters. Electron. J. Combin. Number Theory, 8(A40):1-19, 2008.

[24] T.J. Schaefer. The complexity of satisfiability problems. In Proceedings of the tenth annual ACM symposium on Theory of computing, STOC '78, pages 216-226, New York, NY, USA, 1978. ACM.

[25] V. G. Vizing. On an estimate of the chromatic class of a p-graph. Journal of Mathematical Biology, 3:23-30, 1964. (Russian).

[26] P. Zhang. A study on generalized solution concepts in constraint satisfaction and graph colouring. Master's thesis, University of British Columbia, Canada, 2014. 